ANTÔNO ARIIDO REGINAIDO DE HOLANDA ${ }^{1}$

Ana Katherine da SilverRa Gonçalves ${ }^{2}$

TÉcia Maria de Olivera Maranhão²

Artigo Original

Palavras-chave

Gravidez

Ultrassonografia mamária

Fluxometria por laser-doppler Artérias mamárias/ultrassonografia

Keywords

Pregnancy

Ultrasonography, mammary

Laser-doppler, flowmetry Mammary arteries/ultrasonography

\section{Características ultrassonográficas mamárias e índices hemodinâmicos das artérias mamárias internas durante a gravidez normal}

\author{
Breast ultrasonographic features and internal mammary \\ artery bemodynamic indexes during normal pregnancy
}

\section{Resumo}

OBJETIVO: Avaliar as características ultrassonográficas mamárias e os índices hemodinâmicos das artérias mamárias internas em grávidas normais, correlacionando-os com os períodos da gestação. MÉTODOS: Estudo epidemiológico, observacional e transversal, realizado entre agosto de 2013 e fevereiro de 2015, com 93 mulheres distribuídas em três grupos: primeiro trimestre, segundo trimestre e terceiro trimestre. As variáveis dependentes foram as espessuras da pele, do tecido celular subcutâneo, do tecido fibroglandular, do tecido adiposo retromamário, o diâmetro dos ductos, assim como os índices de pulsatilidade e resistência das artérias mamárias internas. As variáveis independentes foram os três períodos da gestação. Para a análise estatística, empregou-se o teste de Levene (variâncias uniformes entre os períodos da gestação), o teste ANOVA com medidas repetidas, o teste de Tukey de comparação múltipla e de contraste. O teste $t$ de Student foi utilizado para avaliar a diferença entre nulíparas e não nulíparas, e o coeficiente de correlação de Pearson para a correlação entre as duas mamas. Foi considerado o nível de significância de 5\%. RESULTADOS: A média de idade foi $26,6 \pm 4,6$ anos, a qual não houve diferença significativa entre os grupos. A localização da mama (direita/esquerda) e o período gestacional não tiveram efeito significativo sobre as espessuras mamárias da pele, tecido celular subcutâneo e tecido adiposo retromamário, porém a espessura do tecido fibroglandular e o diâmetro dos ductos apresentaram diferença significativa em relação ao período gestacional $(p<0,001)$ do primeiro para o terceiro e do segundo para o terceiro trimestres. A dopplerfluxometria das artérias mamárias internas revelou diferença entre as mamas e o período gestacional, ou seja, o lado direito apresentou medidas superiores ao lado esquerdo, e os valores foram decrescentes ao longo da gestação $(p<0,001)$. CONCLUSÃO: A espessura média de tecido fibroglandular e o diâmetro dos ductos mostraram diferenças significativas do primeiro para o segundo e do primeiro para o terceiro trimestre, não sendo observadas diferenças entre as duas mamas. $\bigcirc$ índice de pulsatilidade e o índice de resistência das artérias mamárias internas foram progressivamente menores durante a gravidez.

\section{Abstract}

PURPOSE: To evaluate breast ultrasonographic features and hemodynamic indexes of the internal mammary arteries in normal pregnant women, and their correlation with the gestational periods. METHODS: Observational and crosssectional, epidemiological, study, conducted between August 2013 and February 2015, with 93 women divided into three groups: first trimester, second trimester and third trimester. The dependent variables were thickness of the skin, of subcutaneous tissue, fibroglandular tissue, and retrommamary adipose tissue, the diameter of the ducts, as well as the pulsatility and resistance indexes of the internal mammary arteries. Independent variables were the three periods of gestation. Repeated measures ANOVA with the multiple comparison Tukey test and a test of contrasts were used for statistical analysis. The Levene test was used to test the homogeneity of variances between periods of gestation. Student's ttest was used to evaluate the difference between nulliparous and non -nulliparous women, and Pearson's correlation coefficient was used for correlation analysis between the two breasts. The level of significance was set at $5 \%$. RESULTS: Mean age was $26.6 \pm 4.6$ years, with no significant difference among groups. Breast location (right/left) and gestational period had no significant effect on the thickness of the skin, of subcutaneous tissue and
Correspondêncio

Antônio Arildo Reginaldo de Holanda Rua Joaquim Câmara, 226/201 - Tirol CEP: $59015-270$

Natal (RN), Brasil

Recebido

$28 / 04 / 2015$
Maternidade Escola Januário Cicco, Departamento de Tocoginecologia da Universidade Federal do Rio Grande do Norte - UFRN Natal (RN), Brasil.

'Programa de Pós-graduação (Doutorado) em Ciências da Saúde, Universidade Federal do Rio Grande do Norte - UFRN - Natal (RN), Brasil.

2Departamento de Tocoginecologia, Universidade Federal do Rio Grande do Norte - UFRN - Natal (RN), Brasil.

Conflito de interesses: não há. 
adipose retromammary tissue. However, the thickness of fibroglandular tissue and the diameter of the ducts showed a significant difference according to gestational period $(p<0.001)$, i.e., from the first to the second and to the third trimesters. Doppler flowmetry of the internal mammary arteries showed a difference between breasts and between gestational periods, i.e., the measurements of the right breast were greater than those of the left, and these values decreased throughout pregnancy $(p<0.001)$. CONCLUSION: The average thickness of fibroglandular tissue and the diameter of the ducts showed significant differences from the first to the second and to the third trimesters, with no differences being observed between the two breasts. The pulsatility and resistance indexes of the internal mammary arteries decreased progressively throughout pregnancy.

\section{Introdução}

Durante a gravidez e lactação, várias mudanças fisiológicas que são atribuídas às alterações hormonais ocorrem nas mamas e causam hiperplasia vascular e lobular. As alterações nos níveis séricos de progesterona, estrogênio e prolactina são os fatores responsáveis por essas mudanças, que se iniciam no primeiro trimestre da gravidez ${ }^{1}$. Durante o primeiro trimestre, sob a influência do estrogênio, ocorre a proliferação ductal e, em menor grau, crescimento lobular. Isso é acompanhado por involução do estroma fibrogorduroso, bem como por um aumento da vascularização e do fluxo sanguíneo ${ }^{1,2}$. Durante o segundo e o terceiro trimestres, a progesterona induz hiperplasia lobular, assim como contínua involução do estroma fibrogorduroso. Por causa da ação estrogênica, a proliferação ductal se torna muito intensa ${ }^{1,3}$. A influência da prolactina na produção láctea é inibida por níveis elevados do estrogênio e da progesterona até o final da gravidez, quando as células alveolares produzem colostro sob ação da prolactina ${ }^{1,2,4}$.

Tais mudanças fisiológicas manifestam-se clinicamente pelo aumento de volume das mamas, associado ao entumescimento e ao aumento da nodularidade ${ }^{5}$, o que reduz progressivamente a acurácia do exame clínico ${ }^{6,7}$ bem como do diagnóstico por imagem, retardando frequentemente a detecção precoce de enfermidades ${ }^{7}$. Não obstante, a sensibilidade ultrassonográfica para detecção de lesões é de $95,2 \%$ e do exame clínico, de $66,7 \%{ }^{8}$. Apesar da importância da avaliação mamária no ciclo grávido-puerperal, poucos estudos investigando a anatomia das mamas têm sido conduzidos desde que Cooper estudou as mamas lactantes com dissecções há mais de 160 anos $^{9,10}$. Ademais, inconsistências na avaliação da anatomia das mamas no ciclo grávido-puerperal entre alguns estudos têm sido observadas ${ }^{11}$.

A avaliação mamária no ciclo grávido-puerperal por ultrassonografia requer um conhecimento sólido da anatomia, das patologias mamárias e das técnicas de imagem exclusivas, sobretudo quando se tratar de mamas lactantes ${ }^{11}$. Ainda que a mamografia ${ }^{5,6}$ e também a ressonância magnética ${ }^{12}$ sejam necessárias e complementares à ultrassonografia para alguns diagnósticos no ciclo grávido-puerperal ${ }^{7}$, a ultrassonografia constitui o método radiológico mais apropriado para avaliar os distúrbios das mama em mulheres durante a gravidez e a lactação $0^{5,6,12,13}$ por conferir maior sensibilidade ${ }^{3,14}$, na faixa de 86,7 a $100 \%{ }^{3}$ quando comparada a $30 \%$ na mamografia ${ }^{14}$. Dados mais recentes, todavia, mostram uma pequena vantagem da mamografia $(80,9 \%)$, em comparação à ultrassonografia (77\%), na detecção de cânceres na gravidez e puerpério ${ }^{15}$.

O fluxo sanguíneo mamário tem sido bem estudado em animais, porém em mulheres os dados são ainda escassos, admitindo-se que este aumente, principalmente por meio de ramos da artéria mamária interna (AMI) e ramos da artéria torácica lateral (ATL), as quais fornecem 60-70\% e $30 \%$ desse fluxo, respectivamente ${ }^{8,16}$. Alguns estudos em animais documentam uma relação positiva do fluxo sanguíneo com a produção láctea, mas em mulheres não há informações seguras nesse sentido ${ }^{16}$. Entretanto, a redução substancial desse fluxo em mulheres lactantes com baixa produção sugere um limiar abaixo do qual a produção de leite fica comprometida ${ }^{7}$.

Durante a gravidez, as mamas podem ser afetadas por uma variedade de desordens específicas, como processos inflamatórios, infecciosos, papilomatose juvenil e também tumores benignos ou malignos ${ }^{5,6}$. Tais mudanças fisiológicas também retardam o diagnóstico de doenças, como o câncer ${ }^{17}$. Apesar disso, as diversas publicações relativas às mamas no ciclo grávido-puerperal abordam praticamente apenas doenças concomitantes ${ }^{1,3,5,7,18}$, sendo ainda insuficiente o número de estudos sobre alterações fisiológicas, anatômicas e funcionais mamárias nessa fase. É necessária, todavia, uma melhor compreensão da anatomia mamária e das mudanças fisiológicas a fim de mais prontamente diagnosticar anormalidades ${ }^{8}$ e otimizar a abordagem das doenças ${ }^{3}$.

Nesse sentido, o presente estudo teve como objetivo avaliar e quantificar as alterações das mamas durante a gravidez normal, por meio de ultrassonografia mamária com dopplerfluxometria das artérias mamárias internas, correlacionando-as com os períodos da gravidez.

\section{Métodos}

Este estudo, classificado como epidemiológico, observacional e transversal, foi realizado na cidade de Natal, Rio Grande do Norte, Brasil, na Maternidade Escola Januário Cicco, da Universidade Federal do Rio Grande do Norte (UFRN), com mulheres encaminhadas pelo próprio serviço ou de Unidades Básicas de Saúde para exame ultrassonográfico de rotina no Setor de Imagem 
entre agosto de 2013 e fevereiro de 2015 . A pesquisa foi conduzida de acordo com a declaração de Helsinque, revisada em 2008, sendo previamente cadastrada na Plataforma Brasil (PLBR) e analisada pelo Comitê de Ética em Pesquisa do Hospital Universitário Onofre Lopes, da UFRN (CEP/HUOL - UFRN), recebendo parecer favorável (número 369.467 - CAAE: 17598813.4.0000.5292). Todas as mulheres participantes assinaram o Termo de Consentimento Livre e Esclarecido (TCLE).

Participaram do estudo grávidas clinicamente normais, com idades entre 19 e 35 anos, distribuídas em três grupos: primeiro trimestre, segundo trimestre e terceiro trimestre. Para cada uma foi preenchida uma ficha clínica de atendimento, compreendendo dados epidemiológicos e clínicos, e os achados do exame a que foram submetidas (ultrassonografia mamária com dopplerfluxometria das artérias mamárias internas). Foi ainda avaliado o Índice de Massa Corporal (IMC) de todas as mulheres, tendo como referência de normalidade a tabela de Atalah-Samur et al. ${ }^{19}$, usada na gravidez.

Como critérios de exclusão, foram definidos a presença de doenças mamárias, endócrinas, hepáticas, renais, cardiovasculares, obesidade (IMC variando desde 30,1 na $6^{\text {a }}$ semana a 33,3 na $42^{\text {a }}$ semana, segundo a tabela proposta por Atalah-Samur et al. ${ }^{19}$ ) e outras quaisquer comorbidades que implicassem um aumento do risco gestacional. Como covariáveis, foram consideradas: idade, paridade, sinais gravídicos e simetria mamária. Como variável independente, foi considerado o período gestacional (primeiro, segundo e terceiro trimestres), e como variáveis dependentes, as espessuras da pele, do tecido celular subcutâneo, do tecido fibroglandular, do tecido adiposo retromamário, o diâmetro dos ductos, assim como os Índices de pulsatilidade (IP) e resistência (IR) das artérias mamárias internas (AMI).

À temperatura ambiente de $25^{\circ} \mathrm{C}$, as mulheres foram submetidas à ultrassonografia mamária com dopplerfluxometria das AMI, pelo mesmo examinador, utilizando equipamento dinâmico (GE, Logiq, Little Chalfont, Reino Unido) em modo bidimensional, empregando-se uma sonda linear, na frequência de 7 a $10 \mathrm{MHz}$, estando as mulheres em decúbito dorsal, com as mãos atrás da cabeça. As mamas foram examinadas desde a linha paraesternal até a linha axilar média e da clavícula ao sulco inframamário nos planos transversal, longitudinal, radial (transdutor em corte transversal e oblíquo, sentido horário) e antirradial (transdutor em corte transversal e oblíquo, sentido anti-horário). As espessuras foram avaliadas empregando-se a média de três medidas nos quadrantes superiores. A medição do tecido fibroglandular não incluiu áreas contendo gordura em permeio. AMI foram escolhidas para avaliação por serem responsáveis por 60 a $70 \%$ do fluxo sanguíneo mamário e também em razão de a agenesia das ATL ocorrer entre 16 e $18 \%$ das mulheres ${ }^{16}$. A identificação das AMI baseou-se em técnica documentada, na qual se posiciona o transdutor em plano transversal, a partir do esterno, entre o segundo e o sexto espaços intercostais ${ }^{16}$.

A avaliação estatística consistiu em apresentação dos eventos em números absolutos, percentuais, médias e desvios padrão para as covariáveis. As variáveis dependentes (espessuras da pele, do tecido celular subcutâneo, do tecido fibroglandular, do tecido adiposo retromamário e diâmetro dos ductos) foram exploradas usando gráficos Box-Plot e histogramas (checar normalidade), e o teste de Levene foi usado para conferir a hipótese de variâncias uniformes do erro entre os períodos de gestação (grupos independentes).

Verificada a distribuição normal, foi aplicado o teste ANOVA com medidas repetidas tendo como fatores a variável período da gestação, e a localização da mama (lado direito e esquerdo) como fator de repetição. Rejeitada a hipótese nula de igualdade entre as médias dos três grupos, foi aplicado o teste de Tukey de comparação múltipla para identificar onde estão as diferenças. Para avaliar a correlação entre as duas mamas, de acordo com cada variável dependente, foi calculado o coeficiente de correlação de Pearson. O teste $t$ de Student foi utilizado para avaliar a diferença entre as médias de nulíparas e não nulíparas em cada variável dependente nos dois lados (direito e esquerdo). Foi utilizado um nível de significância de $5 \%$.

\section{Resultados}

Participaram do estudo 93 grávidas, distribuídas em três grupos: $1^{\circ}, 2^{\circ}$ e $3^{\circ}$ trimestre, compostos respectivamente por 31,32 e 30 mulheres, cuja média

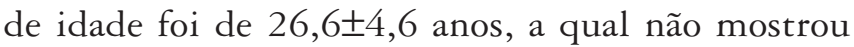
diferença significativa entre os grupos. As idades ges-

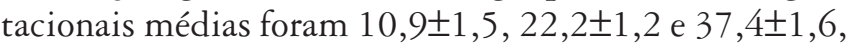
respectivamente para $1^{\circ}, 2^{\circ}$ e $3^{\circ}$ trimestres. As nulíparas representaram $38,7 \%$ da amostra. No exame clínico das mamas, foi observada simetria em $96 \%$ e a presença de sinais gravídicos em $92,5 \%$.

A Tabela 1 mostra que a lateralidade (direita/esquerda) e o período gestacional não tiveram efeito significativo sobre a média das espessuras mamárias: pele, tecido celular subcutâneo e tecido adiposo retromamário. A espessura do tecido fibroglandular, apesar de não ter apresentado diferença significativa quanto à localização $(p=0,9)$, mostrou diferença significante com relação ao período gestacional $(\mathrm{p}<0,0001)$. As espessuras, respectivamente, para o $1^{\circ}, 2^{\circ}$ e $3^{\circ}$ trimestres foram $1,2 \pm 0,2,1,4 \pm 0,3$

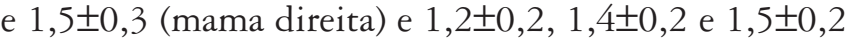
(mama esquerda). O teste de comparações múltiplas de Tukey revelou diferença significativa entre as médias do 
$1^{\circ}$ e $2^{\circ}$ trimestres $(p<0,0001)$ e entre as médias do $1^{\circ}$ e $3^{\circ}$ trimestres $(\mathrm{p}=0,01)$. Da mesma maneira, o diâmetro dos ductos apresentou diferenças significativas com relação ao período gestacional $(\mathrm{p}<0,001)$, mas não quanto à localização da mama $(\mathrm{p}=0,6)$, sendo, respectivamente, as medidas no $1^{\circ}, 2^{\circ}$ e $3^{\circ}$ trimestres: 0,2 $\pm 0,06,0,27 \pm 0,05$ e 0,29 $\pm 0,06$ (mama direita) e 0,22 $\pm 0,05,0,27 \pm 0,04$ e $0,28 \pm 0,04$ (mama esquerda). Nessa variável, o teste de comparações múltiplas de Tukey, também revelou diferença significativa entre as médias do $1^{\circ}$ e $2^{\circ}$ trimestres $(\mathrm{p}<0,0001)$ e entre as médias do $1^{\circ}$ e $3^{\circ}$ trimestres $(\mathrm{p}<0,01)$.

A Tabela 2 mostra os resultados observados à dopplerfluxometria das AMI. Observou-se efeito do lado e do período gestacional, ou seja, o lado direito teve medidas sempre superiores ao lado esquerdo $(\mathrm{p}=0,001)$, decrescendo o valor com o progredir da gravidez $(\mathrm{p}<0,001)$. Os valores do IP, respectivamente, para o $1^{\circ}, 2^{\circ}$ e $3^{\circ}$ trimestres

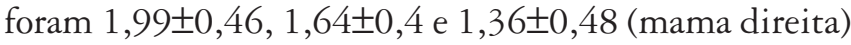

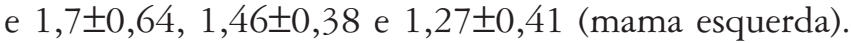

Os valores do IR respectivamente para o $1^{\circ}, 2^{\circ} \mathrm{e} 3^{\circ}$ trimestres foram $85 \pm 0,08,0,77 \pm 0,12$ e $0,68 \pm 0,14$ (mama direita), e $0,77 \pm 0,1,0,71 \pm 0,09$ e $0,66 \pm 0,11$ (mama esquerda). O teste de comparações múltiplas de Tukey revelou que os três períodos são significativamente diferentes dois a dois, ou seja, houve diferença do IP quando se comparou as médias do $1^{\circ}$ e $2^{\circ}$ trimestres $(\mathrm{p}=0,01)$, do $1^{\circ}$ e $3^{\circ}$ trimestres $(\mathrm{p}<0,001)$ e do $2^{\circ}$ e $3^{\circ}$ trimestres $(\mathrm{p}=0,03)$. Para o IR, essas diferenças foram do $1^{\circ}$ e $2^{\circ}$ trimestres $(\mathrm{p}=0,007)$, do $1^{\circ}$ e $3^{\circ}$ trimestres $(\mathrm{p}<0,001)$ e do $2^{\circ}$ e $3^{\circ}$ trimestres $(\mathrm{p}=0,006)$.

A associação das variáveis dependentes com a nuliparidade mostrou haver diferença significativa entre nulíparas (idade média de $24,8 \pm 4,1$ ) e não nulíparas (idade média de 27,7 $\pm 4,4$ ) quanto à espessura do tecido celular subcutâneo no lado direito $(\mathrm{p}=0,004)$ e no esquerdo $(\mathrm{p}=0,003)$, sendo a média da espessura em nulíparas de $0,6 \pm 0,2$ (mama direita) e 0,6 $\pm 0,2$ (mama esquerda) e em não nulíparas $0,79 \pm 0,29$ (mama direita) e $0,78 \pm 0,28$

Tabela 1. Média e desvio padrão das medidas ultrassonográficas mamárias segundo o período da gestação e a mama (direita/esquerda)

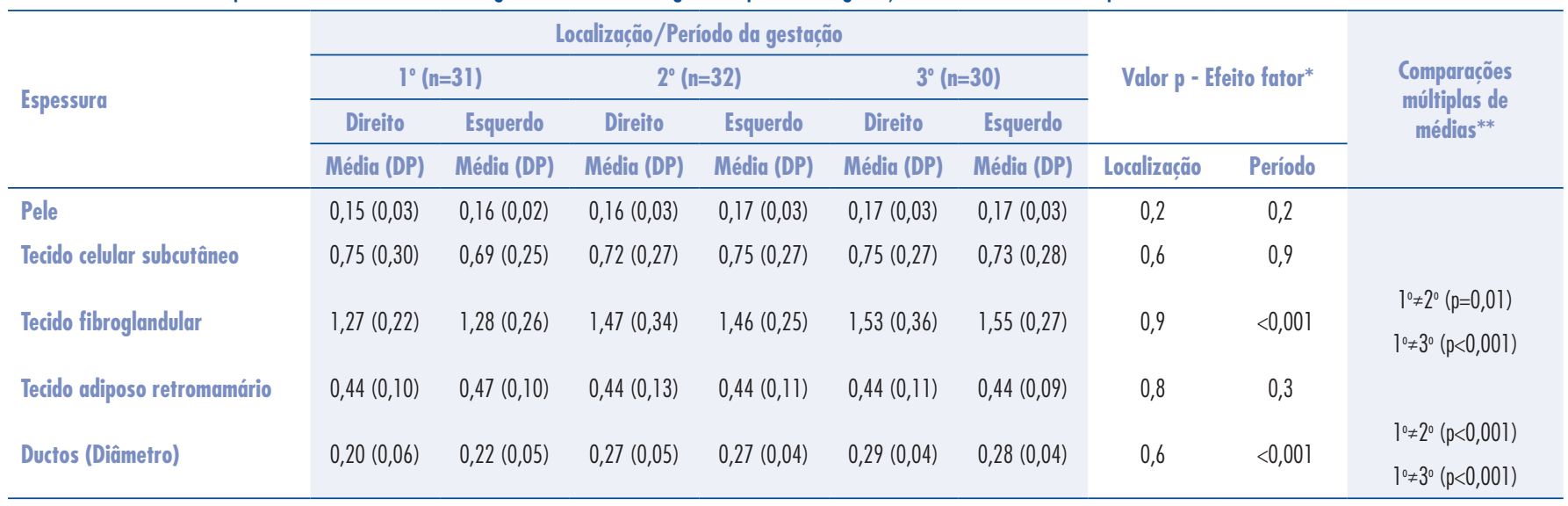

*Teste ANOVA de medidas repetidas avaliando o efeito da localização e período; * ${ }^{*}$ Teste de Tukey mostrando onde estão as diferenças de médias entre períodos.

DP: desvio padrão.

Tabela 2. Média e desvio padrão de índice de pulsatilidade e resistência das artérias mamárias internas de acordo com os fatores período da gestação e a localização da mama

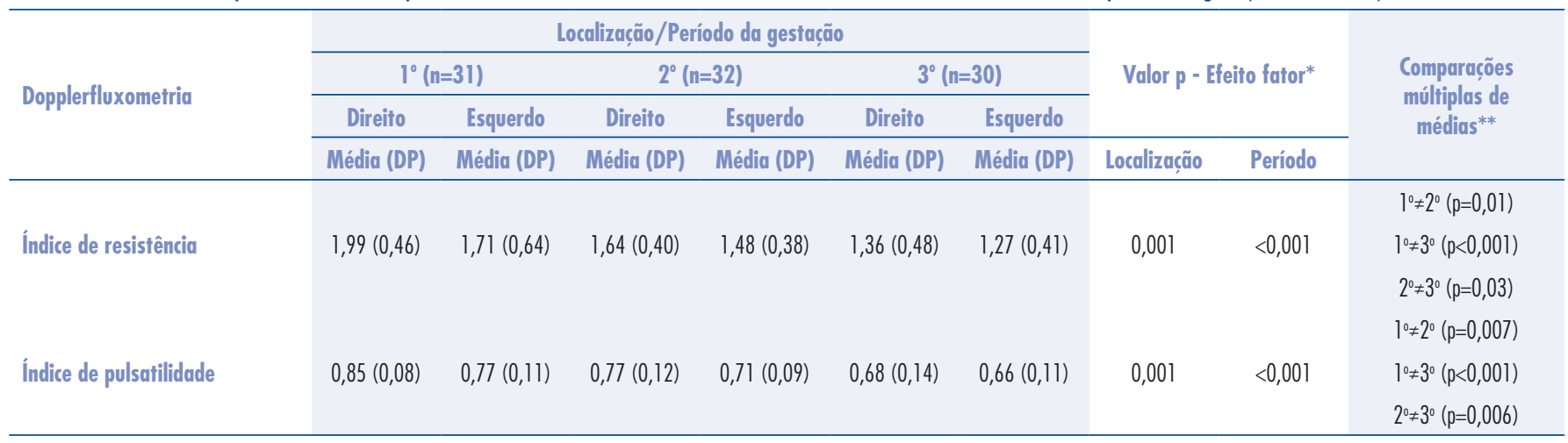

*Teste ANOVA de medidas repetidas avaliando o efeito da localização e período; **Teste de Tukey mostrando onde estão as diferenças de médias entre períodos.

DP: desvio padrão. 
(mama esquerda), mostrando semelhança. Com relação às demais medidas e aos resultados dos IP e IR, não houve diferenças significativas.

A correlação das medidas ultrassonográficas entre as duas mamas, avaliada por meio do teste de correlação de Pearson, mostrou diferença significante $(\mathrm{p}<0,0001)$. As espessuras dos tecidos celular subcutâneo, fibroglandular e o diâmetro dos ductos apresentaram correlações respectivamente de $0,7,0,7$ e 0,8 , classificadas como fortes $(r>7,0)$.

\section{Discussão}

A ultrassonografia para avaliação das mamas alcançou enormes avanços técnicos nos últimos anos, melhorando consideravelmente a resolução das imagens e permitindo inclusive o estudo de pequenas estruturas ${ }^{10}$. No entanto, o seu emprego requer um conhecimento sólido da anatomia e das doenças mamárias, bem como de técnicas de imagem exclusivas, especialmente durante a lactação $0^{11,16}$, pela maior frequência de diagnósticos quando comparada com a gravidez ${ }^{18,20}$. Esse método, por ser considerado apropriado para a avaliação mamária no ciclo grávido-puerperal ${ }^{21}$ e acessível, foi empregado neste estudo.

As modificações fisiológicas dos tecidos mamários na gravidez são motivadas por alterações endócrinas, metabólicas e imunológicas ${ }^{21}$. Com relação à pele, tais alterações ocorrem em $90 \%$ das grávidas ${ }^{22,23}$, que se tornam também propensas a apresentarem dermatoses específicas ${ }^{22}$. O presente estudo mostrou que houve aumento gradativo da espessura da pele ao longo da gravidez, porém tais diferenças não foram significativas.

Não foram também observadas diferenças significativas entre os demais tecidos mamários quando comparadas as mama direita e esquerda, sendo, porém, encontradas mudanças na espessura do tecido fibroglandular e no diâmetro dos ductos quando comparados os três períodos da gravidez. Esses dados estão em sintonia com um estudo realizado com lactantes, que também não revelou diferenças dos tecidos adiposo e fibroglandular na mesma mulher, encontrando, todavia, diferenças entre mulheres distintas ${ }^{10}$. Esses resultados sugerem um padrão de simetria mamária, o que está de acordo com a observação clínica do presente estudo, pois $96 \%$ das mamas apresentaram-se simétricas. A literatura refere que em lactantes ocorre um predomínio do tecido fibroglandular sobre o adiposo, na razão de 2:1, e em não lactantes, de 1:1 ${ }^{10}$, parecendo que, quando ocorre assimetria mamária, esta se relaciona com a redução do tecido fibroglandular ${ }^{10}$.

Apesar da avaliação do fluxo sanguíneo mamário em animais ser bem documentada na literatura ${ }^{16}$, os dados em mulheres no ciclo grávido-puerperal são ainda escassos. Ainda assim, os resultados dos estudos com animais não são unânimes, havendo controvérsias se o fluxo sanguíneo interfere na produção láctea ${ }^{16}$.

O presente estudo revelou que os índices de pulsatilidade e de resistência das AMI apresentaram diferenças significativas tanto em relação à localização mamária (lado direito com medidas superiores ao lado esquerdo) quanto ao período gestacional, em que os valores foram decrescentes com o progredir da gestação. Não encontramos estudos abordando avaliação dopplerfluxométrica das AMI na gestação, porém dados relativos às artérias uterinas mostram igualmente tendência ao decréscimo, inversamente proporcional à idade gestacional ${ }^{24}$.

Com este estudo, foi possível também avaliar se havia diferenças entre nulíparas e não nulíparas quanto às mensurações. Observou-se que houve diferença significativa entre esses dois grupos quanto à espessura do tecido celular subcutâneo em ambas as mamas, sendo a média da espessura em nulíparas semelhante. Não obstante a distribuição heterogênea de tecido adiposo e fibroglandular na mama dificultar a medição desses tecidos, a proporção de tecido fibroglandular e adiposo estimado pela mamografia é 1:1 em média, havendo alteração dessa relação com o avançar da idade, por causa do declínio do tecido fibroglandular e maior proporção do tecido adiposo?.

Os resultados deste estudo possibilitaram caracterizar numericamente, mediante a mensuração dos tecidos mamários, as mudanças que ocorrem na gravidez, assim como a dinâmica dos índices hemodinâmicos das artérias mamárias internas, permitindo concluir que a espessura média do tecido fibroglandular e o diâmetro dos ductos não diferem quando comparadas as mamas de uma mesma mulher, sendo, porém, influenciados pelo período da gravidez, com diferença significativa do primeiro para o segundo e do primeiro para o terceiro trimestre. Os índices de pulsatilidade e de resistência das artérias mamárias internas foram progressivamente menores ao longo da gravidez, com valores mais elevados do lado direito, sendo tais diferenças significativas entre todos os períodos gestacionais entre si. Tais dados possibilitam maior compreensão das mudanças fisiológicas relacionadas com os períodos da gestação, servindo de parâmetros de comparação quando grávidas normais precisarem ser avaliadas por ultrassonografia mamária.

\section{Agradecimentos}

Ao Professor Dr. António Manuel Gouveia de Oliveira, da Universidade de Lisboa, Professor Visitante da UFRN, pela revisão da análise estatística. 
1. Yu JH, Kim M, Cho H, Liu HJ, Han SJ, Ahn TG. Breast diseases during pregnancy and lactation. Obstet Gynecol Sci. 2013;56(3):143-59.

2. Canoy JM, Mitchell GS, Unold D, Miller V. A radiologic review of common breast disorders in pregnancy and the perinatal period. Semin Ultrasound CT MR. 2012;33(1):78-85.

3. Joshi S, Dialani V, Marotti J, Mehta TS, Slanetz PJ. Breast disease in the pregnant and lactating patient: radiological-pathological correlation. Insights Imaging. 2013;4(5):527-38.

4. Neville MC. Anatomy and physiology of lactation. Pediatr Clin North Am. 2001;48(1):13-34.

5. Vashi R, Hooley R, Butler R, Geisel J Philpotts L. Breast imaging of the pregnant and lactating patient: imaging modalities and pregnancy-associated breast cancer. AJR Am J Roentgenol. 2013;200(2):321-8.

6. Sabate JM, Clotet M, Torrubia S, Gomez A, Guerrero R, de las Heras $P$, et al. Radiologic evaluation of breast disorders related to pregnancy and lactation. Radiographics. 2007;27(Suppl 1):S101-24.

7. Langer A, Mohallem M, Chérel P. [Pregnancy associated breast cancer: A review of the literature]. Imagerie Femme. 2013;23(2):1168. French.

8. Ezeonu PO, Ajah LO, Onoh RC, Lawani LO, Enemuo VC, Agwu UM. Evaluation of clinical breast examination and breast ultrasonography among pregnant women in Abakaliki, Nigeria. Onco Targets Ther. 2015;8:1025-9.

9. Geddes DT. Inside the lactating breast: the latest anatomy research. J Midwifery Womens Health. 2007;52(6):556-63.

10. Ramsay DT, Kent JC, Hartmann RA, Hartmann PE. Anatomy of the lactating human breast redefined with ultrasound imagining. J Anat. 2005;206(6):525-34.

11. Geddes DT. Ultrasound imaging of the lactating breast: methodology and application. Int Breastfeed J. 2009;4:4.

12. Kalogerakos K, Sofoudis C, Tzonis P, Koutsouradis P, Katsoulis $G$. Breast cancer and pregnancy; overview of international bibliography. J BUON. 2013;18(2):308-13.

13. Taylor D, Lazberger J, Ives A, Wylie E, Saunders C. Reducing delay in the diagnosis of pregnancy-associated breast cancer: how imaging can help us. J Med Imaging Radiat Oncol. $2011 ; 55(1): 33-42$.

14. Ohta T, Okamoto K, Kanemaki Y, Tsujimoto F, Nakajima Y, Fukuda $M$, et al. Use of ultrasonography as an alternative modality for first-line examination in detecting breast cancer in selected patients. Clin Breast Cancer. 2007;7(8):624-6.

15. Langer A, Mohallem M, Stevens D, Rouzier R, Lerebours F, Chérel $P$. A single-institution study of 117 pregnancy-associated breast cancers $(\mathrm{PABC})$ : presentation, imaging, clinicopathological data and outcome. Diagn Interv Imaging. 2014;95(4):435-41.

16. Geddes DT, Aljazaf KM, Kent JC, Prime DK, Spatz DL, Gardin CP, et al. Blood flow characteristics of the human lactating breast. J Hum Lact. 2012;28(2): 145-52.

17. Lee SS, Hartman HJ, Kuzmiak CM, Crosby KL. Management of breast symptoms in the pregnant and lactating patient. Curr Obstet Gynecol Rep. 2013;2(1):53-8.

18. Ulery M, Carter L, McFarlin BL, Giurgescu C. Pregnancy-associated breast cancer: significance of early detection. J Midwifery Womens Health. 2009;54(5):357-63.

19. Atalah-Samur E, Castillo-L C, Castro-Santoro R, Aldea-P A. [Proposal of a new standard for the nutritional assessment of pregnant women]. Rev Med. Chil. 1997;125(12):1429-36. Spanish.

20. Ohta T, Okamoto K, Kanemaki Y, Tsujimoto F, Nakajima Y, Fukuda $M$, et al. Use of ultrasonography as an alternative modality for first-line examination in detecting breast cancer in selected patients. Clin Breast Cancer. 2007;7(8):624-6.

21. Zerouali A, Zaraa I, Trojiet S, El Euch D, Azeiez MI, Mokni M, et al. [Physiologic skin changes in pregnancy]. Presse Med. 201 1;40(1 Pt 1):e17-21. French.

22. Kar S, Krishnan A, Shivkumar PV. Pregnancy and skin. J Obstet Gynaecol India. 2012;62(3):268-75.

23. Vora RV, Gupta R, Mehta MJ, Chaudhari AH, Pilani AP, Patel N. Pregnancy and skin. J Family Med Prim Care. $2014 ; 3(4): 318-24$

24. Costa AG, Spara P, Costa TO, Tejo Neto WR. Uterine arteries resistance and pulsatility indices at the first and second trimesters of normal pregnancies. Radiol Bras. 2010;43(3):161-5. 